\title{
COVID-19 and Physical Activity: How Can We Build Back Better?
}

\author{
Catherine E. Draper, Karen Milton, and Jasper Schipperijn
}

COVID-19 has disrupted and impacted almost everyone's life around the world, and with that also our physical activity behavior and environments that influence physical activity. There has been a proliferation of publications in 2020 on COVID-19 and physical activity, covering a wide range of topics, including the impact of lockdowns and COVID-19 on physical activity levels and behaviours amongst adults, ${ }^{1,2}$ children, and adolescents ${ }^{3}$; the consequences of inactivity $^{4-6}$; the need to stay active while in quarantine ${ }^{7}$ (included in the official recommendations of some countries ${ }^{8}$ ) for both physical and mental health; the role of physical activity in immunity against SARS-CoV-29-12; and how to exercise safely after recovering from COVID-19. ${ }^{13,14}$

In spite of all this new information, no one knows exactly how COVID-19 will continue to influence our lives in years to come, but we feel that now is a good time to reflect on what COVID-19 can teach us in terms of changes to physical activity behavior and environments. Hopefully this can lead the way to a series of positive developments for the field of physical activity and health and help us to 'build back better'. In this editorial, we highlight some of the key learnings for the physical activity and health field arising from COVID-19.

\section{The Need for Intersectoral Collaboration and Systems Approaches}

The COVID-19 pandemic has, perhaps more than any other public health crisis in recent years, highlighted the importance of intersectoral collaboration and systems approaches, which have been recently acknowledged by the International Society for Physical Activity and Health (ISPAH) in its 'Eight Investments that work for physical activity' (https://www.ispah.org/resources/key-resources/ 8 -investments/). The importance of these approaches is applicable both from a global perspective, for international agencies such as the World Health Organization (WHO) and the United Nations Children's Fund (UNICEF), but also within countries. While country responses have varied widely, it has been clear that public health alone will not get us through this pandemic. Multiple government departments have had to rally their resources to respond to COVID-19, and multiple scientific disciplines have been called on for their expertise. It is essential to continue applying this approach in the field of physical activity and health, particularly with regards to creating enabling environments for physical activity - in the broadest sense.

\footnotetext{
Draper is with the SAMRC/Wits Developmental Pathways for Health Research Unit, Faculty of Health Sciences, University of the Witwatersrand, Johannesburg, South Africa; and the Division of Exercise Science and Sports Medicine, Department of Human Biology, University of Cape Town, Cape Town, South Africa. Milton is with the Norwich Medical School, University of East Anglia, Norwich, United Kingdom. Schipperijn is with the Department of Sports Science and Clinical Biomechanics, University of Southern Denmark, Odense, Denmark. Draper (Catherine.Draper@wits.ac.za) is corresponding author.
}

\section{Research Translation and Effective Science Communication Are More Important Than Ever}

While we would have hoped that a crisis such as COVID-19 would have the whole world turning to science for answers, this has unfortunately not been the case. With social media's ability to amplify 'fake news', and some leading governments appearing to not 'follow the science', the scientific community has been forced to face the uncomfortable reality that science does not have the final word. Furthermore, the constantly changing landscape of the science of COVID-19 has made the communication of clear and credible public health recommendations far more difficult. What we can take from this for the field of physical activity and health is that how we translate our research and communicate our scientific findings is more critical than ever. With the recent release of the 2020 WHO guidelines on physical activity and sedentary behaviour (https://apps.who.int/iris/bitstream/handle/10665/336656/ 9789240015128-eng.pdf), we have the opportunity to do better at this, on a global level. Locally, we need to consider how our messages about physical activity and sedentary behaviour are communicated in a way that takes contextual realities into consideration, and perhaps with a greater recognition of conflicting messages or broader narratives that could conflict with our efforts to promote physical activity and provide enabling environments.

\section{Don't Leave Out Mental Health}

It could be argued that research on the benefits of physical activity has historically favored those relating to physical health, such as the prevention of non-communicable diseases and weight management. While there is convincing evidence that physical activity is beneficial for mental health across a range of age groups, COVID-19 appears to have helped these mental health benefits take their rightful place alongside the physical health benefits. While this looks different across global settings, there are certainly some common behavioral threads: the importance of active play for children's social and emotional health (particularly in the face of rising screen time in lockdown); and the value of doing recreational activity (especially being out in nature) to take a break from the home environment. Given the COVID-19 experiences of 2020 (and beyond), we are reminded that mental health should not be left out of the picture, and we are hopeful that individuals, families, communities, and policy makers will be more receptive to hearing about the mental health benefits of physical activity — so long as we communicate it effectively, of course.

\section{Keep Physical Activity on the Priority Agenda}

In many high-income countries, COVID-19 has provided the opportunity for the promotion of active transportation, by creating 
new bike lanes, open streets, and improved pedestrian infrastructure to create more liveable environments. Many of these might be temporary adjustments, but it is essential to consider how these developments might be made more permanent. Despite many of these positive improvements, it is possible that across many countries-at all income levels - physical activity may have fallen even further down the priority list, both at an individual and a government level. In the midst of more pressing economic concerns and the need for resources to be allocated to COVID-19, how will physical activity fare when it comes to policy decisions and budget allocations? Globally, COVID-19 and lockdown restrictions have amplified inequalities; food insecurity and hunger are topics that have come up in some of the world's leading economies, and these issues have worsened in many low- and middle-income countries. Encouraging an unemployed and anxious mother of hungry children to go outside and do some exercise-in an environment that is unappealing and potentially unsafe-seems even more unrealistic than before.

However, these challenges are not a reason to throw in the towel and wait until COVID-19 has passed before we get back to advocating for physical activity. ISPAH's 'Eight Investments that work for physical activity' can help policy and decision makers to prioritize those strategies that are most likely to be successful, which makes sense in these difficult economic times. Furthermore, now is also a good time to reflect on how to create long term physical activity behavior changes to help countries achieve the recently updated WHO physical activity and sedentary behavior guidelines for all age groups and for pregnant and postpartum women, adults living with chronic conditions, and children and adults living with disability (https://www.who.int/publications/i/ item/9789241550536). These guidelines are more clear and evidence-based than ever before, and can be used in conjunction with ISPAH's 'Eight Investments that work for physical activity' to help achieve the WHO's global target to reduce physical inactivity by $15 \%$ by 2030 (https://www.who.int/ncds/prevention/physicalactivity/global-action-plan-2018-2030/en/).

\section{Conclusion}

Our learnings from COVID-19 provide us with key actions for the field of physical activity and health: continue pursuing intersectoral collaboration and the application of systems approaches; strive to translate our research and communicate our science effectively for both policymakers and the public; ensure that the mental health benefits of physical activity are part of the picture; and keep physical activity on the priority agenda, both locally and internationally. We have more resources than ever before with evidencebased guidelines on physical activity and sedentary behaviors across the life course, priority strategies for action with the 'Eight Investments', and a global action plan on physical activity to help realize the goal of 'more active people for a healthier world'. Physical activity can play a significant role in building back better as we navigate post-pandemic realities-for individuals, families, schools, workplaces, communities, nations, and across the globe.

$J S$ is the current President of the International Society for Physical Activity and Health (ISPAH); KM is President Elect of ISPAH; CED is the Past President of ISPAH.

\section{References}

1. Matsungo TM, Chopera P. Effect of the COVID-19-induced lockdown on nutrition, health and lifestyle patterns among adults in Zimbabwe. BMJNPH. 2020;28. doi:10.1136/bmjnph-2020-000124

2. Chopra S, Ranjan P, Singh V, et al. Impact of COVID-19 on lifestylerelated behaviours - a cross-sectional audit of responses from nine hundred and ninety-five participants from India. Diabetes Metab Syndr. 2020;14(6):2021-2030. PubMed ID: 33099144 doi:10.1016/ j.dsx.2020.09.034

3. Bates LC, Zieff G, Stanford K, et al. COVID-19 impact on behaviors across the 24-hour day in children and adolescents: physical activity, sedentary behavior, and sleep. Children. 2020;7(9):138-139. PubMed ID: 32947805 doi:10.3390/children7090138

4. Arora T, Grey I. Health behaviour changes during COVID-19 and the potential consequences: a mini-review. J Health Psychol. 2020; 25(9):1155-1163. PubMed ID: 32551944 doi:10.1177/13591053 20937053

5. Lesser IA, Nienhuis CP. The impact of COVID-19 on physical activity behavior and well-being of Canadians. Int J Environ Res Public Health. 2020;17(11):3899. PubMed ID: 32486380 doi:10.3390/ ijerph17113899

6. Ingram J, Maciejewski G, Hand CJ. Changes in diet, sleep, and physical activity are associated with differences in negative mood during COVID-19 lockdown. Front Psychol. 2020;11:588604. PubMed ID: 32982903 doi:10.3389/fpsyg.2020.588604

7. World Health Organization. \#HealthyAtHome-physical activity. World Health Organization. https://www.who.int/news-room/campaigns/ connecting-the-world-to-combat-coronavirus/healthyathome/healthyat home---physical-activity. Published 2020. Accessed January 13, 2021.

8. Public Health Scotland. Coronavirus (COVID-19): physical activity. https://www.nhsinform.scot/illnesses-and-conditions/infections-andpoisoning/coronavirus-covid-19/healthy-living/coronavirus-covid19-physical-activity. Published 2020. Accessed January 13, 2021.

9. Chastin S, Abaraogu U, Bourgois J, et al. Physical activity, immune function and risk of community acquired infectious disease in the general population: systematic review and meta-analysis. SSRN. 2020. doi:10.2139/ssrn.3673184

10. Jesus I, Vanhee V, Deramaudt TB, Bonay M. Promising effects of exercise on the cardiovascular, metabolic and immune system during COVID-19 period. J Hum Hypertens. 2021;35(1):1-3. PubMed ID: 32943741 doi:10.1038/s41371-020-00416-0

11. Ranasinghe C, Ozemek C, Arena R. Exercise and well-being during COVID 19-time to boost your immunity. Expert Rev Anti Infect Ther. 2020;18(12):1195-1200. PubMed ID: 32662717 doi:10.1080/ 14787210.2020 .1794818

12. da Silveira MP, da Silva Fagundes KK, Bizuti MR, Starck É, Rossi $\mathrm{RC}$, de Resende e Silva DT. Physical exercise as a tool to help the immune system against COVID-19: an integrative review of the current literature [published online ahead of print July 29, 2020]. Clin Exp Med. 1-14. PubMed ID: 32728975 doi:10.1007/s10238-02000650-3

13. Salman D, Vishnubala D, Le Feuvre P, et al. Returning to physical activity after covid-19. BMJ. 2021;372:m4721. PubMed ID: 33419740 doi:10.1136/bmj.m4721

14. Kennedy FM, Sharma S. COVID-19, the heart and returning to physical exercise. Occup Med (Lond). 2020;70(7):467-469. PubMed ID: 32816003 doi:10.1093/occmed/kqaa154 